\title{
Cancer stem-neuroendocrine cells in an atypical carcinoid case report
}

\author{
Valentina Masciale ${ }^{1 \#}$, Giulia Grisendi ${ }^{2 \#}$, Federico Banchelli ${ }^{3}$, Roberto D'Amico ${ }^{3}$, Antonino Maiorana ${ }^{4}$, \\ Uliano Morandi ${ }^{1}$, Massimo Dominici ${ }^{2 *}$, Beatrice Aramini $^{1 *}$ \\ ${ }^{1}$ Division of Thoracic Surgery, ${ }^{2}$ Division of Oncology, ${ }^{3}$ Center of Statistic, ${ }^{4}$ Institute of Pathology, Department of Medical and Surgical Sciences, \\ University of Modena and Reggio Emilia, Modena, Italy \\ \#These authors contributed equally to this work as co-first authors. \\ *These authors contributed equally to this work as co-last authors. \\ Correspondence to: Beatrice Aramini, MD, PhD. Division of Thoracic Surgery, Department of Medical and Surgical Sciences, University of Modena \\ and Reggio Emilia, Via Largo del Pozzo, 71, 41124, Modena, Italy. Email: beatrice.aramini@unimore.it.
}

\begin{abstract}
Lung neuroendocrine cells tumor (NET) classification and diagnosis, particularly for typical and atypical carcinoids, are complicated by a variable natural history and nonspecific symptoms. Mechanisms for the development and progression of well-differentiated lung NETs are still unclear. An accurate and timely diagnosis can ensure the implementation of appropriate treatment and impact on prognosis. One of the main unclear point is the definition of these cells' composition. In fact, it is known that carcinoids are mainly constituted by neuroendocrine cells. Aim of our report is to show for the first time the presence of a high percentage of cancer stem cells (CSCs) in an atypical carcinoid. The ALDEFLUOR assay was used to identify and sort $\mathrm{ALDH}^{\text {high }}$ and $\mathrm{ALDH}^{\text {low }}$ human lung cancer cells following tissue digestion. SOX2 was additionally determined by immunohistochemistry. All specimens contained the $53.10 \%$ of ALDHhigh cells among all viable lung cancer cells, which indicates that more than half of the entire tumor cell population was composed by CSCs. As expected also in immunohistochemistry, about a half of the nuclei of the cells were positive for SOX2. We strongly support the hypothesis of the presence of cancer stem-neuroendocrine cells (CSCs-NETs) as subpopulation in these types of tumors.
\end{abstract}

Keywords: Cancer stem cells (CSCs); neuroendocrine cells tumor (NETs); atypical carcinoid; carcinoid tumors (TCs); case report

Submitted Nov 04, 2019. Accepted for publication Nov 15, 2019.

doi: $10.21037 /$ tlcr.2019.12.07

View this article at: http://dx.doi.org/10.21037/tlcr.2019.12.07

\section{Introduction}

Although lung neuroendocrine tumors (NETs) are considered a distinct family of lung tumors (1), there is evidence suggesting that typical carcinoid tumors (TCs) and atypical carcinoid tumors (ACs) are morphologically distinct from large-cell neuroendocrine carcinomas (LCNECs) and small-cell lung cancer (SCLC) (1). Lung NETs can be classified as well-differentiated (low-grade typical carcinoids and intermediate-grade atypical carcinoids) and poorly differentiated (high-grade large-cell neuroendocrine carcinoma or SCLC) (2). The World Health Organization
(WHO) classification of lung NETS was updated in 2015 and organized the types of lung NETs on a spectrum (3). However, the most important point of differentiation analysis is the dichotomous distinction between tumors that are low-grade (typical carcinoid and atypical carcinoid) and high-grade (large-cell neuroendocrine and small-cell carcinomas) (4,5). In particular, with regards of low- and intermediate-grade NETS, the differences are mainly based on the number of mitoses (2).

The staging of lung NETs is based on the TNM staging of non-NET lung cancers, which adheres to the current WHO classification system (6). Given that many 


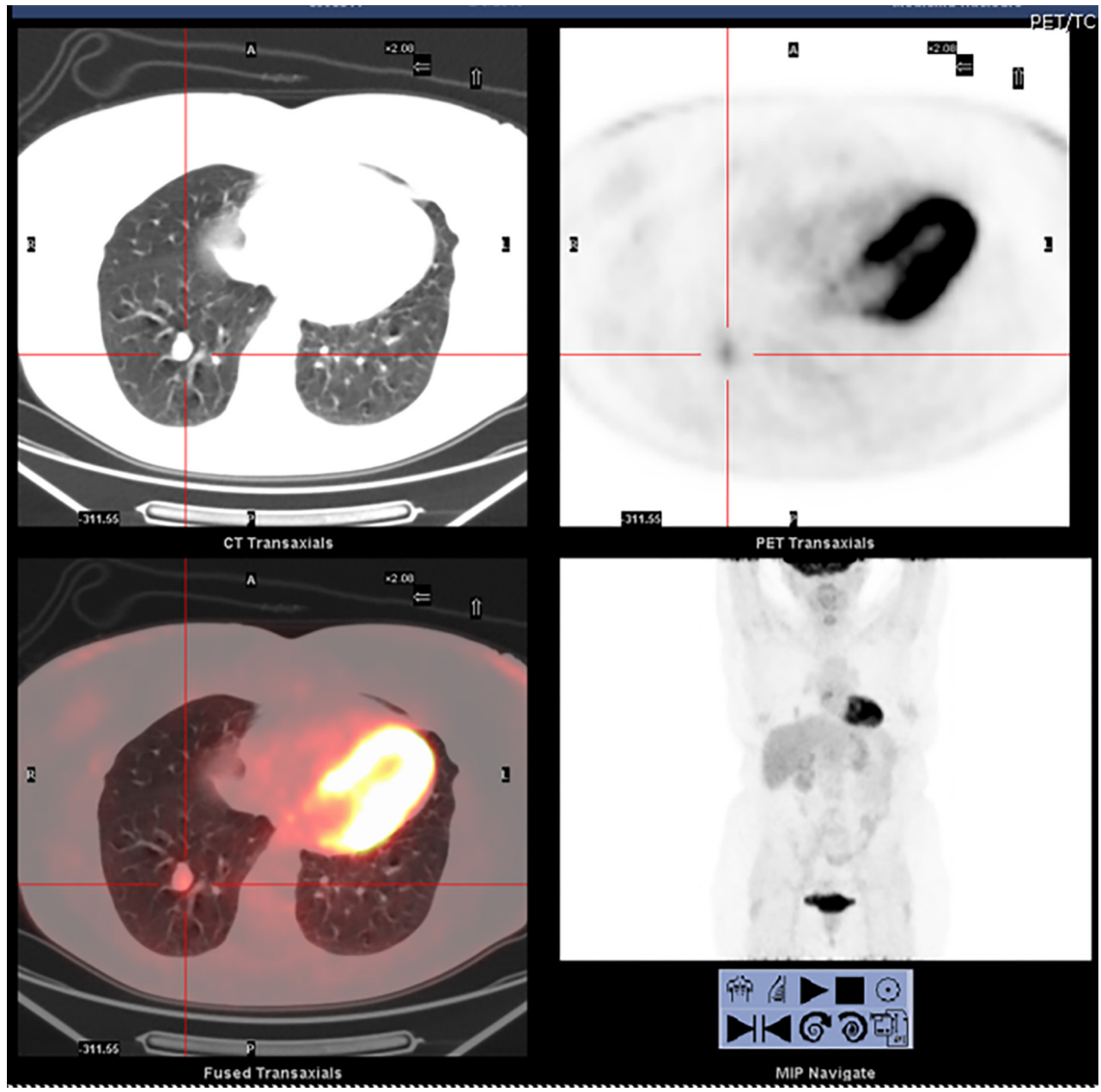

Figure 1 Lesion of the right lower lobe. ${ }^{18} \mathrm{~F}-\mathrm{FDG}$ PET showed a mild hypermetabolic nodule $(\mathrm{SUVmax}=2.5) .{ }^{18} \mathrm{~F}-\mathrm{FDG}$ PET, ${ }^{18} \mathrm{~F}$-fluorodesossiglucose positron emission tomography.

lung carcinoids and atypical carcinoids are larger than $3 \mathrm{~cm}$, this staging system is not ideal for this subset of lung malignancies (7). In fact, $5 \%$ to $20 \%$ of TCs and $30 \%$ to $40 \%$ of ACs metastasize, and many patients present with recurrent disease or metastases to the liver or bone (8). As a result, delays in diagnosis increase the probability of developing metastatic disease, which has a negative impact on prognosis (8). One of the main points that remains unclear is cell composition of these tumors.

Our report suggests that there is a large cancer stem cells (CSCs) subpopulation in carcinoid tumors. We speculate that this elevated percentage of CSCs may be related to the aggressiveness of the tumor, as represented by the characteristics of this intermediate grade tumor which tends to metastasize.

This new finding represents an important aspect in the definition of future markers even for neuroendocrine tumors with diagnostic and therapeutic purposes.

\section{Methods}

\section{Case presentation}

We present a case of 58-year-old woman who came to our attention for a chest $\mathrm{X}$-ray accidental finding, due to recurrent fever episode, of a $2 \mathrm{~cm}$ nodule at the level of the basal segment of the right lower lobe of the lung. Chest CT with enhancement confirmed the presence of a solid formation in the right lower lobe and a ${ }^{18} \mathrm{~F}$-fluorodesossiglucose positron emission tomography $\left({ }^{18} \mathrm{~F}-\mathrm{FDG}\right.$ PET) showed a mild hypermetabolic lesion (SUVmax 2.5), very suspicious for carcinoid tumor (Figure 1). Preoperative assessment and pulmonary function test 
were in normal ranges. In February 2018, the patient was undergone right lower lobectomy by lateral thoracotomy. The intraoperative histological examination described cells highly suspicious for neuroendocrine tumor, which was confirmed later by the diagnosis of atypical carcinoid of the lung, with mitotic activity equal to 2 for $10 \mathrm{HPF}$. Immunohistochemistry was positive for chromogranin, CD56, synaptophysin and negative for TTF1.

\section{Cells extraction and fluorescence-activated cell sorting (FACS) analysis}

Tumor tissue was washed in sterile Dulbecco's PBS (L1825BC, Merck Millipore) and mechanically minced into small pieces (2 to $4 \mathrm{~mm}$ ), further digested using a tumor dissociation kit in a disposable gentle MACS $^{\mathrm{TM}} \mathrm{C}$-Tube (Miltenyi) according to the manufacturer's instructions. Samples were digested for $60 \mathrm{~min}$ at $37^{\circ} \mathrm{C}$ in a gentle MACS Octo dissociator and filtered through $70-\mu \mathrm{m}$ sterile cell strainers, centrifuged at $300 \times \mathrm{g}$ for $5 \mathrm{~min}$, and resuspended in a DMEM and HAM'S F12 media mixture (2:1) (Gibco) containing $50 \mathrm{IU} / \mathrm{mL}$ penicillin-streptomycin and $4 \mathrm{mM}$ glutamine. Single-cell suspensions of the primary tumor cells were diluted in ALDEFLUOR assay buffer containing BODIPY-aminoacetaldehyde (STEMCELL Technologies, Vancouver, BC). Cell morphology was evaluated using side scatter (SSC) and forward scatter (FSC). Dead cells were excluded using 7-AAD (7-amino-actinomycin D) staining. Cell sorting and ALDH analysis were performed using a FACS-ARIA III (Becton Dickinson, Franklin Lakes, NJ). The results were analyzed using FACS Diva software (Becton Dickinson). The gating strategy included the $\mathrm{ALDH}^{\text {high }}$ gate, which was set at least one log apart from the $\mathrm{ALDH}^{\text {low }}$ gate.

\section{Immunobistochemistry}

Patient's slides were deparaffinized, rehydrated and then washed in PBS. Sodium citrate buffer was used for antigen retrieval. Samples were incubated with the anti-SOX2 (1:200) (MA1-014 Thermo Fisher Scientific, Meridian Road Rockford, IL, USA), overnight at $4{ }^{\circ} \mathrm{C}$. Images were collected and the positivity evaluated using a Zeiss Axioskop microscope with a Zeiss AxioCam ICc 3 High-Resolution Microscope Camera.

\section{Results}

\section{Clinical conditions from surgery}

No complications were shown during and after the operation. The patient was discharge from Hospital after 6 days from surgery. A chest X-ray at 6 and 30 days from surgery was negative for lesions or pleural-parenchyma alterations. The oncologist suggested to proceed with monitoring through follow up.

\section{$A L D H^{\text {high }}$ stem cells were identified in primary cells of atypical carcinoid of the lung}

Tumor tissue dissociation efficiently released cancer cells characterized by a heterogeneous morphology, as illustrated in the widespread FSC and SSC values (Figure 2). The mean viability of the samples was $99.7 \%$ based on 7 -AAD staining. These data further confirmed that the developed dissociation procedure was a non-toxic approach to isolating cells from tumor tissues. The CSCs were physically separated from the bulk parental tumor cells and recovered by FACS according to the following gating strategy. Tumor cells were first identified based on their morphological parameters (FSC/ SSC), and ALDH activity was measured in the 7-AAD negative cell population only (Figure 2). $\mathrm{ALDH}^{\text {low }}$ and $\mathrm{ALDH}^{\text {high }}$ cells were both selected and sorted (Figure 2). An $\mathrm{ALDH}^{\text {high }}$ subpopulation was the $53.10 \%$ of all viable lung cancer cells, which indicates that more than half of the entire tumor cell population was composed by CSCs.

\section{Immunobistochemical evaluation of the SOX2 stem cell marker in atypical lung cancer cells}

To further evaluate the stemness of the cells extracted from the tumor biopsy, SOX2 immunohistochemistry was performed. Tissue sections were examined at $10 x$ magnification to characterize the overall staining pattern and at $20 \times$ and $40 \times$ magnification for a more accurate evaluation of the positivity of the cells, represented by brown nuclei. As expected by FACS analysis, about a half of the nuclei of the cells were positive for SOX2 (Figure 3).

\section{Discussion}

In our case, we show the presence of similar percentage 

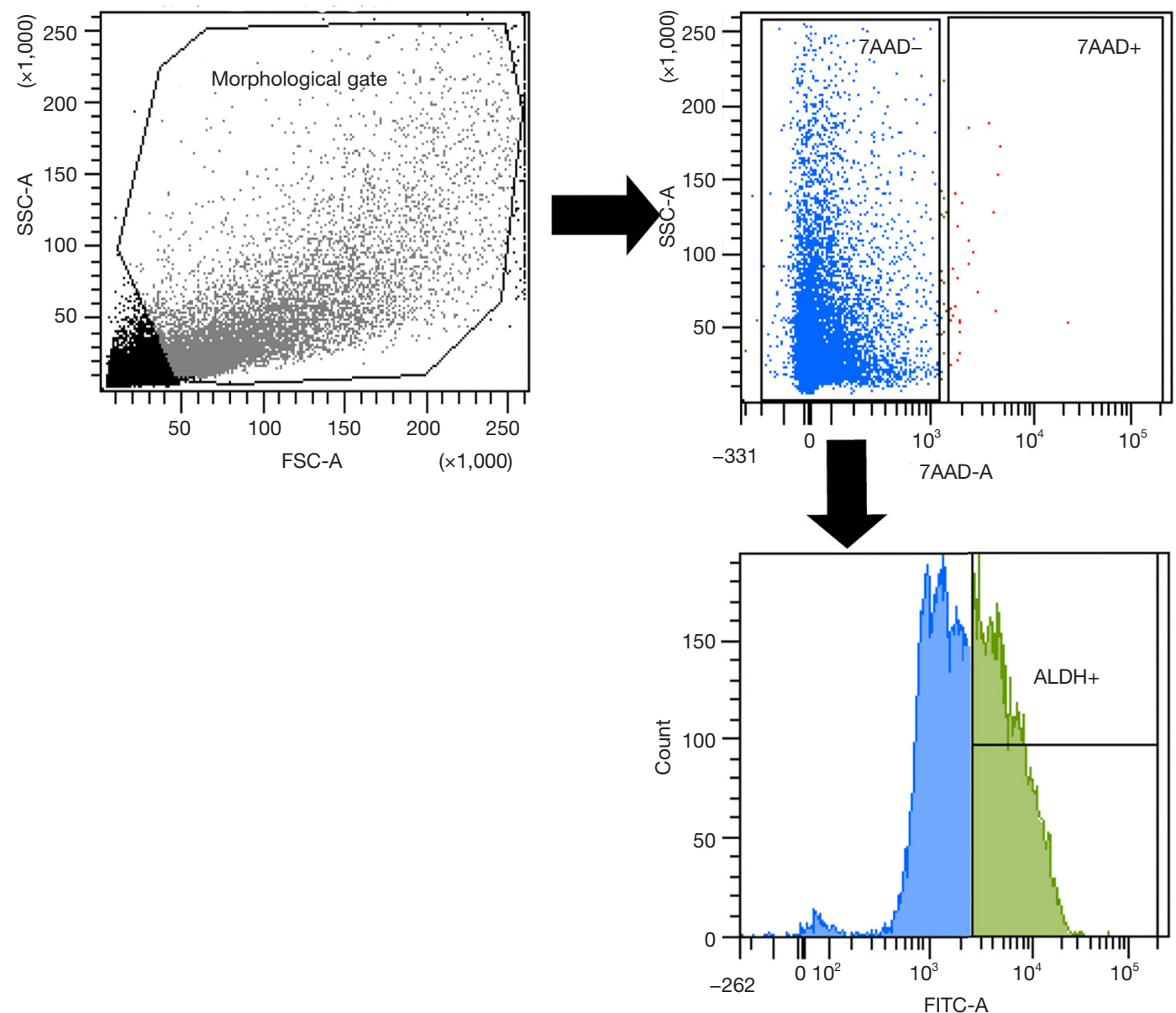

Figure 2 FACS analysis. Cytofluorimetric analysis of ALDH ${ }^{\text {high }}$ cells in atypical carcinoid primary cells. FACS, fluorescence-activated cell sorting.
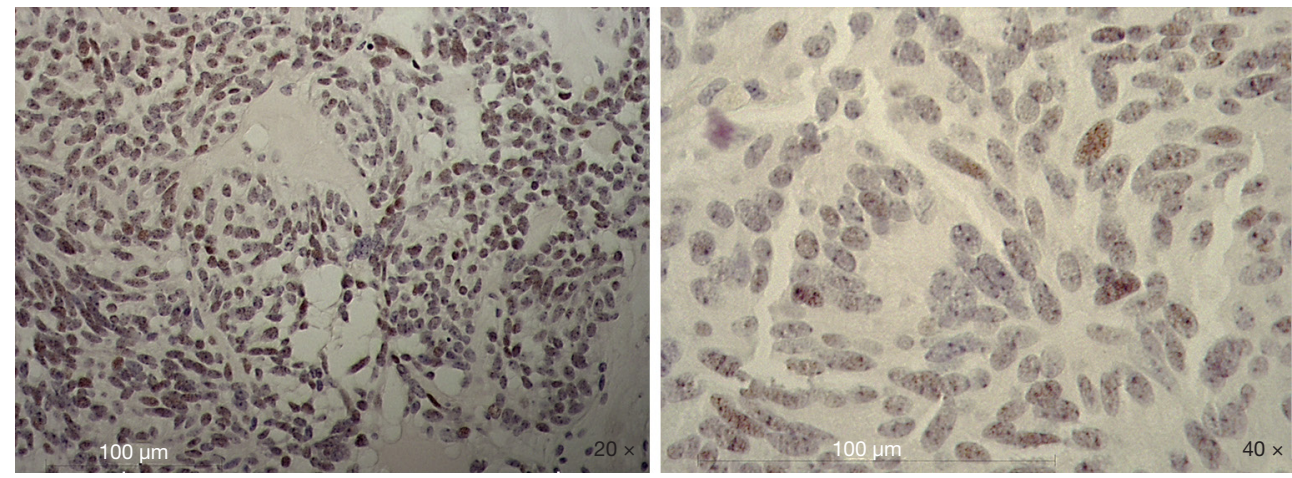

Figure 3 Immunohistochemistry of lung atypical carcinoid. Anti-SOX2 monoclonal antibody of tumor slides. Representative images were shown in $20 \times$ and $40 \times$ magnification. 
of CSCs by cytofluorimetric and immunohistochemistry analysis as first finding in the knowledge of atypical carcinoids tumors. This aspect is very important suggesting a new characterization of neuroendocrine tumors. We used ALDH for cytofluorimetric analysis and SOX2 marker for immunohistochemistry, which are the most representative markers for CSCs (9) and we noted a very high percentage of CSCs comparable between the two techniques. In particular, the $50 \%$ of ALDH positivity through the cytofluorimetric analysis was found. As previously described, aldehyde dehydrogenase (ALDH) is an enzyme that participates in important cellular mechanisms as aldehyde detoxification and retinoic acid synthesis; moreover, ALDH activity is involved in drug resistance, a characteristic of CSCs (10). Even though ALDH is found in stem cells, CSCs and progenitor cells, this enzyme has been successfully used to identify and isolate cell populations with CSC properties from several tumor origins (11). In fact, in 2010 Sullivan et al. identified CSCs in a panel of 11 NSCLC tumor samples, 45 NSCLC lines, and 7 SCLC lines that are used to study ALDH activity and sorted a subpopulation of NSCLC stem-like cells dependent on Notch signaling (12). As previously described, SCLC is a high-grade SCLC which is part of lung NET. However, nobody at this time has previously described the presence of CSCs in carcinoid tumors (13).

As previously described, ALDH+ population in NSCLC is usually not represented by a high percentage of CSCs, which is normally not more than 5\% (12). In particular, Huang et al. (14) analyzed in H28, H2052 lung metastatic cells lines a sub-population of chemoresistant cells by using ALDH as CSC markers and observed an ALDH+ expression in $\mathrm{H} 28$ of $2.29 \% \pm 1.6 \%$, and in $\mathrm{H} 2052$ of $0.82 \pm 0.32$. Moreover, Bayat Mokhtari et al. (15) in bronchial carcinoid cell lines was observed an ALDH+ expression that reach at least $1 \%$.

We share this concept for the first time with this message: if the presence of CSCs will be confirmed at high percentage in more cases, that will be the basement for a new definition of pathological markers as well as new molecular findings for neuroendocrine tumors.

Beside the limitation of a single case, we strongly support the hypothesis of the presence of cancer stemneuroendocrine cells (CSCs-NETs) as subpopulation in these types of tumors. In conclusion, we may speculate that the different aggressiveness of the atypical versus the typical carcinoid could be derived not only from the number of the mitosis but also from the percentage of CSCs. However, translational studies are mandatory in the future to investigate the morphologic cells characterization as well as also the stemness genes recognition.

\section{Acknowledgments}

The Project has been supported by funds from the Division of Thoracic Surgery of the University Hospital of Modena, from the Laboratory of Cellular Therapy of the University of Modena and Reggio Emilia, and from the Italian Ministry of Education, University and Research: Departments of Excellence 2017.

\section{Footnote}

Conflicts of Interest: The authors have no conflicts of interest to declare.

Ethical Statement: The authors are accountable for all aspects of the work in ensuring that questions related to the accuracy or integrity of any part of the work are appropriately investigated and resolved. A written informed consent was obtained from the patient for the publication of this case report and for any images.

\section{References}

1. Hendifar AE, Marchevsky AM, Tuli R. Neuroendocrine Tumors of the Lung: Current Challenges and Advances in the Diagnosis and Management of Well-Differentiated Disease. J Thorac Oncol 2017;12:425-36.

2. Pelosi G, Sonzogni A, Harari S, et al. Classification of pulmonary neuroendocrine tumors: new insights. Transl Lung Cancer Res 2017;6:513-29.

3. Melosky B. Advanced typical and atypical carcinoid tumours of the lung: management recommendations. Curr Oncol 2018;25:S86-93.

4. Rindi G, Klimstra DS, Abedi-Ardekani B, et al. A common classification framework for neuroendocrine neoplasms: an International Agency for Research on Cancer (IARC) and World Health Organization (WHO) expert consensus proposal. Mod Pathol 2018;31:1770-86.

5. Oronsky B, Ma PC, Morgensztern D, et al. Nothing But NET: A Review of Neuroendocrine Tumors and Carcinomas. Neoplasia 2017;19:991-1002.

6. Travis WD, Brambilla E, Burke AP, et al. WHO Classification of Tumours of the Lung, Pleura, Thymus and Heart. 4th edition. Lyon: International Agency for 
Research on Cancer, 2015.

7. Volante M, Gatti G, Papotti M. Classification of lung neuroendocrine tumors: lights and shadows. Endocrine 2015;50:315-9.

8. Yao JC, Hassan M, Phan A, et al. One hundred years after "carcinoid": epidemiology of and prognostic factors for neuroendocrine tumors in 35,825 cases in the United States. J Clin Oncol 2008;26:3063-72.

9. Liu X, Wang L, Cui W, et al. Targeting ALDH1A1 by disulfiram/copper complex inhibits non-small cell lung cancer recurrence driven by ALDH-positive cancer stem cells. Oncotarget 2016;7:58516-30.

10. Clark DW, Palle K. Aldehyde dehydrogenases in cancer stem cells: potential as therapeutic targets. Ann Transl Med 2016;4:518.

11. Toledo-Guzmán ME, Hernández MI, Gómez-Gallegos ÁA, et al. ALDH as a Stem Cell Marker in Solid Tumors.

Cite this article as: Masciale V, Grisendi G, Banchelli F, D'Amico R, Maiorana A, Morandi U, Dominici M, Aramini B. Cancer stem-neuroendocrine cells in an atypical carcinoid case report. Transl Lung Cancer Res 2019;8(6):1157-1162. doi: 10.21037/tlcr.2019.12.07
Curr Stem Cell Res Ther 2019;14:375-88.

12. Sullivan JP, Spinola M, Dodge M, et al. Aldehyde dehydrogenase activity selects for lung adenocarcinoma stem cells dependent on notch signaling. Cancer Res 2010;70:9937-48.

13. Koinis F, Kotsakis A, Georgoulias V. Small cell lung cancer (SCLC): no treatment advances in recent years. Transl Lung Cancer Res 2016;5:39-50.

14. Huang EH, Hynes MJ, Zhang T, et al. Aldehyde dehydrogenase 1 is a marker for normal and malignant human colonic stem cells (SC) and tracks SC overpopulation during colon tumorigenesis. Cancer Res 2009;69:3382-9.

15. Bayat Mokhtari R, Baluch N, Morgatskaya E, et al. Human bronchial carcinoid tumor initiating cells are targeted by the combination of acetazolamide and sulforaphane. BMC Cancer 2019;19:864. 